\title{
A Method for generating marker-less gene deletions in multidrug-resistant Acinetobacter baumannii
}

Ismawati Mohammad Amin ${ }^{1}$, Grace E Richmond ${ }^{3}$, Paromita Sen ${ }^{1}$, Tse Hsien Koh², Laura JV Piddock ${ }^{3}$ and Kim Lee Chua ${ }^{1 *}$

\begin{abstract}
Background: Acinetobacter baumannii is an important nosocomial pathogen that has become increasingly resistant to multiple antibiotics. Genetic manipulation of MDR A. baumannii is useful especially for defining the contribution of each active efflux mechanism in multidrug resistance. Existing methods rely on the use of an antibiotic selection marker and are not suited for multiple gene deletions.

Results: A tellurite-resistant $\left(s_{a c B^{+}}, x y \mid E^{+}\right)$suicide vector, pMo130-Tel ${ }^{R}$, was created for deleting the adeFGH and adelJK operons in two clinical MDR A. baumannii, DB and R2 from Singapore. Using a two-step selection, plasmid insertion recombinants (first-crossover) were selected for tellurite resistance and the deletion mutants (secondcrossover) were then selected for loss of sacB. The DNA deletions were verified by PCR while loss of gene expression in the $\triangle$ adeFGH, $\triangle$ adelJK and $\triangle a d e F G H \triangle$ adelJK deletion mutants was confirmed using qRT-PCR. The contribution of AdeFGH and AdeIJK pumps to MDR was defined by comparing antimicrobial susceptibilities of the isogenic mutants and the parental strains. The deletion of adelJK produced no more than eight-fold increase in susceptibility to nalidixic acid, tetracycline, minocycline, tigecycline, clindamycin, trimethoprim and chloramphenicol, while the deletion of adeL-adeFGH operon alone had no impact on antimicrobial susceptibility. Dye accumulation assays using $\mathrm{H} 33342$ revealed increased dye retention in all deletion mutants, except for the R2 $\triangle$ adeFGH mutant, where a decrease was observed. Increased accumulation of ethidium bromide was observed in the parental strains and all pump deletion mutants in the presence of efflux inhibitors. The efflux pump deletion mutants in this study revealed that only the AdeIJK, but not the AdeFGH RND pump, contributes to antimicrobial resistance and dye accumulation in MDR A. baumannii DB and R2.
\end{abstract}

Conclusions: The marker-less gene deletion method using pMo130-Tel $\left.\right|^{R}$ is applicable for creating single and multiple gene deletions in MDR A. baumannii. The adeFGH and adelJK operons were successfully deleted separately and together using this method and the impact of each efflux pump on antimicrobial resistance could be defined clearly.

Keywords: Acinetobacter baumannii, Multidrug-resistant, AdeFGH, AdelJK, RND pumps, Allelic replacement

\section{Background}

Acinetobacter baumannii, a non-fementing Gramnegative cocco-bacillus, is a frequent cause of nosocomial bloodstream infections and is associated with considerable morbidity and mortality, especially among patients in intensive care or with burns [1]. $A$. baumannii has become increasingly resistant to multiple

\footnotetext{
* Correspondence: bchckl@nus.edu.sg

'Department of Biochemistry, Yong Loo Lin School of Medicine, National University of Singapore, 8 Medical Drive, Singapore 117597, Singapore Full list of author information is available at the end of the article
}

antibiotics, including imipenem and meropenem, the carbapenems of choice for treating multidrug resistant (MDR) A. baumannii infections. The incidence of carbapenem-resistant $A$. baumannii in the United States and Europe is around 54\% and 16\%, respectively, while the incidence in the Asia/Pacific rim is about $80 \%$ [2]. A. baumannii possesses a variety of intrinsic and acquired resistance determinants, including $\beta$-lactamases, class $\mathrm{D}$ oxacillinases, aminoglycoside-modifying enzymes, outer membrane proteins and active efflux systems [3]. Among its intrinsic resistance determinants, overexpression of

\section{Ciomed Central}


the chromosomally encoded active efflux systems of the resistance-nodulation and division (RND) family, such as AdeABC, AdeFGH and AdeIJK pumps, are a mechanism of resistance to a number of antibiotics [4].

The impact of RND pumps to antibiotic resistance in A. baumannii has been demonstrated by inactivating the genes that encode the efflux pumps and the method for gene inactivation involves insertion of an antibiotic resistance gene to select mutants [5-7]. Studies using mutants in which RND efflux pump genes have been inactivated have suggested significant overlap in antibiotics that are substrates of the A. baumannii pumps. For instance, derivatives of the MDR clinical isolate BM4454 in which ade $A B C$ was inactivated had increased susceptibility to the same antibiotics (fluoroquinolones, chloramphenicol, tetracycline, tigecycline and erythromycin) as inactivation of adeIJK in the same isolate [6]. When both adeABC and adeIJK were inactivated in BM4454, increased susceptibility to ticarcillin, previously not observed in the $\triangle a d e A B C$ mutant or the $\triangle$ adeIJK mutant, was seen [6]. Furthermore, overexpression of a pump gene did not always result in an increase in the MIC of the same antibiotics that had increased activity in the pump inactivated mutants. For example, inactivation of ade $A B C$ in the MDR clinical isolate BM4454 did not affect its susceptibility to imipenem, amikacin and cotrimoxazole, but overexpressing adeABC in a nonMDR clinical isolate BM4587 increased the MIC of these antibiotics [4]. Therefore, it is possible that inactivation of a gene by inserting an antibiotic-resistance gene may affect the antimicrobial susceptibility of the pump geneinactivated mutants, thus complicating the interpretation of the results.

To address this possibility and to define clearly the impact of each efflux pump on antibiotic resistance, we propose that genes encoding efflux pumps be deleted using a marker-less strategy first described by Hamad et al (2009) for Burkholderia spp. [8]. The suicide vector, pMo130 was modified to carry a tellurite resistance cassette, a non-antibiotic selection marker [9]. The $A$. baumannii isolates we have tested, including MDR isolates, were sensitive to tellurite and can be counterselected in LB medium containing 30-60 mg/L tellurite. Gene deletion by allelic replacement was selected using a modification of the two-step process described by Hamad et al (2009) [8]. In this study, the adeFGH and adeIJK operons were deleted separately and together in two MDR A. baumannii strains, DB and R2. The adeIJK deletion mutant showed increased susceptibility to nalidixic acid, chloramphenicol, trimethoprim, tetracycline, tigecycline, minocycline and clindamycin, but the deletion of adeL-adeFGH operon had no impact on antimicrobial susceptibility in the two MDR isolates. Genetic and gene expression analyses revealed that the allelic replacement in both MDR strains had occurred. The marker-less gene deletion method we describe is robust and, unlike the creation of mutants by inserting an antibiotic resistance gene, is suitable for deleting multiple genes in MDR A. baumannii.

\section{Results}

Deletion of the A. baumannii adeFGH and adelJK operons

To ensure reproducibility of the method, gene deletions were created for the adeFGH and adeIJK operons, separately and together, in two clinical MDR A. baumannii isolates, DB and R2. A suicide vector harboring a tellurite-resistance marker was first created by inserting a $3.26 \mathrm{~kb} X m a I$-digested tellurite-resistance cassette from pwFRT-Tel ${ }^{\mathrm{R}}$ into the XmaI site of pMo130 to give $\mathrm{pMo} 30-\mathrm{Tel}^{\mathrm{R}}[8,10]$. In addition to the tellurite-resistance marker, pMo130-Tel ${ }^{\mathrm{R}}$ also carries a kanamycin-resistance marker, the reporter gene $x y l E$ which converts pyrocathechol to a yellow-colored 2-hydroxymuconic semialdehyde, and a modified $s a c B$ gene [8]. Next, DNA fragments of approximately $1 \mathrm{~kb}$ upstream and $1 \mathrm{~kb}$ downstream of the target region to be deleted was ligated with linearized pMo130-TelR give pMo130-TelR(Up/Down) (Figure 1A).

To construct the suicide plasmid for deletion of adeFGH, a $1 \mathrm{~kb}$ DNA fragment located upstream of adeF was amplified from R2 genomic DNA using the primer pair: AdeGUp(Not1)F and AdeGUp(BamHI)R (Figure 1B). The amplimer was digested using Not 1 and BamHI and inserted into pMo130-Tel ${ }^{\mathrm{R}}$, creating $\mathrm{pMo} 130-\mathrm{Tel}^{\mathrm{R}}$-adeFGH(UP). Next, another $1 \mathrm{~kb}$ fragment located downstream of adeG was amplified using the primer pair: AdeGDwn(BamHI)F and AdeGDwn(Sph1)R and cut with BamHI and SphI, and inserted into pMo130-Tel ${ }^{\mathrm{R}}$-adeFGH(Up), thus creating pMo130-Tel ${ }^{\mathrm{R}}$ adeFGH(Up/Down) (Figure 1B). The plasmid construct was first introduced in E. coli S17-1 and subsequently delivered into $A$. baumannii $\mathrm{R} 2$ and $\mathrm{DB}$ by biparental conjugation. A. baumannii transconjugants (first crossovers) were selected on LB agar containing $30 \mathrm{mg} / \mathrm{L}$ tellurite and $25 \mathrm{mg} / \mathrm{L}$ gentamicin. These telluriteresistant colonies which carry genomic insertion of pMo130-Tel ${ }^{\mathrm{R}}$-adeFGH (Up/Down) produced yellow colonies when sprayed with $0.45 \mathrm{M}$ pyrocathechol and a $2 \mathrm{~kb}$ amplimer corresponding to the size of the ligated Up and Down DNA fragments with the primer pair: AdeGUp(Not1)F and AdeGDwn(Sph1)R, but did not produce any amplimer with the outward-facing primer pair: pMo130Tel F and pMo130Tel R (Figure 1A) (data not shown). A. baumannii $\mathrm{R} 2$ and $\mathrm{DB}$ harboring the inserted pMo130-Tel ${ }^{\mathrm{R}}$-adeFGH (Up/Down) construct was cultured in LB broth containing 10\% sucrose and passaged daily to select for deletion of adeFGH operon and loss of the $\operatorname{sacB}$ gene by a second cross-over and allelic 
Amin et al. BMC Microbiology 2013, 13:158

Page 3 of 14

http://www.biomedcentral.com/1471-2180/13/158

A

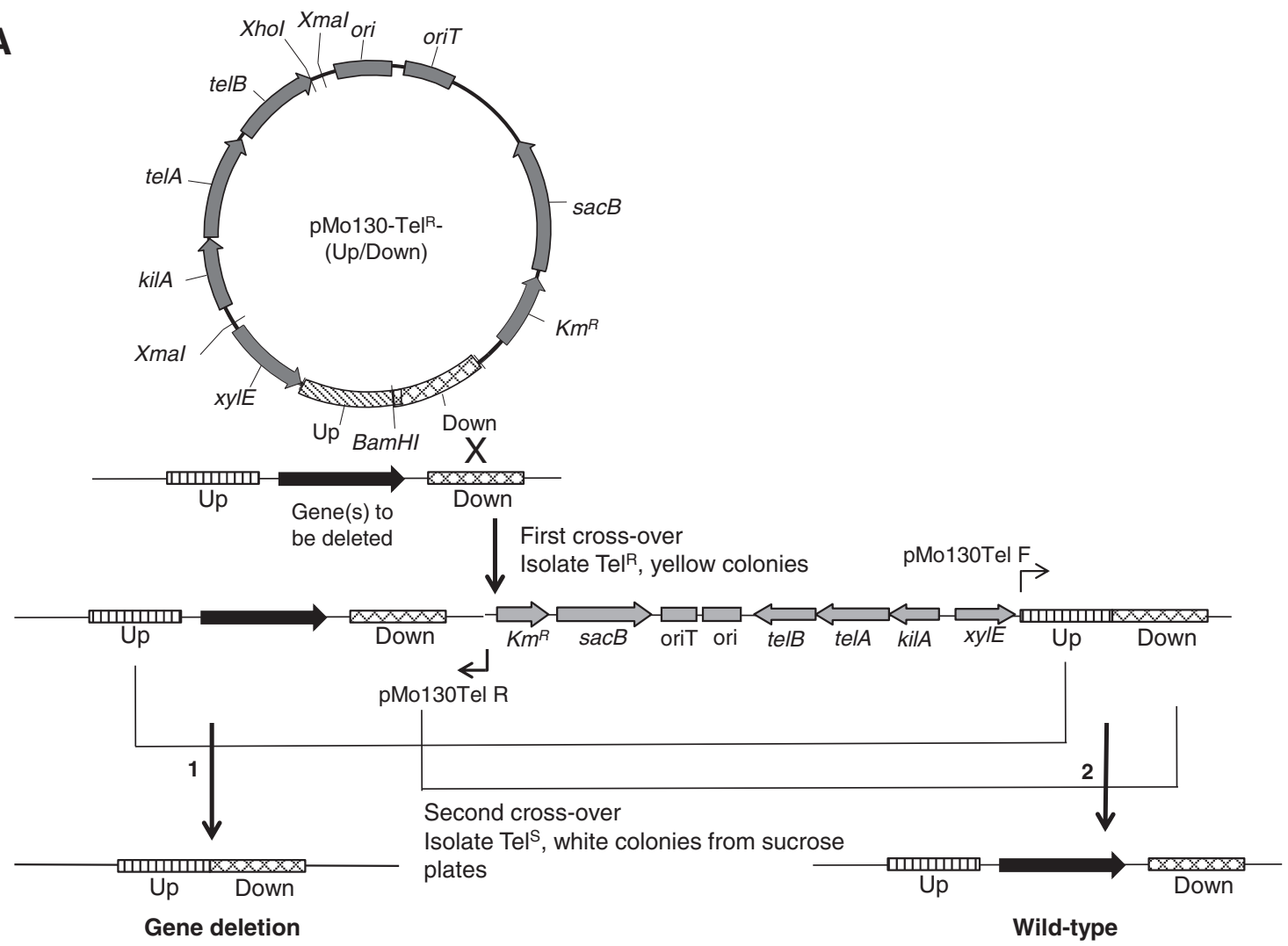

B
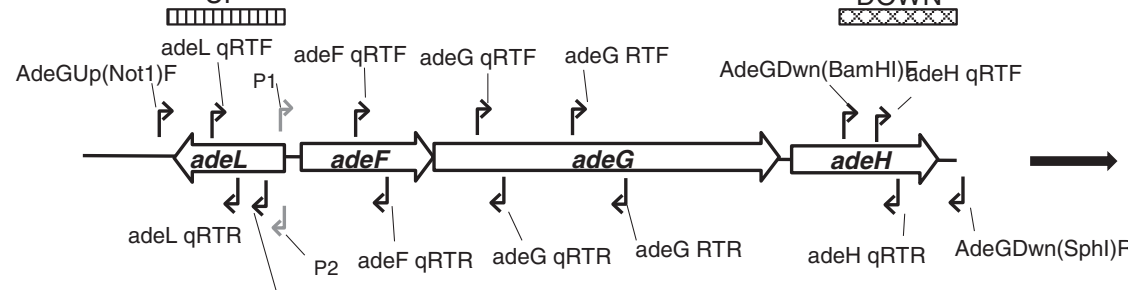

DOWN

DXXXX

ild-type

C AdeGUp(BamHI)R

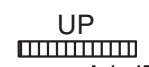

adel qRTF AdeJF

AdeJ(UP) Pstl F

P3

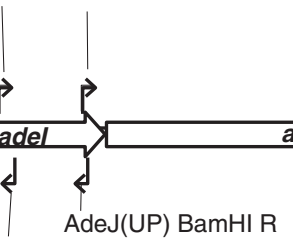

adel qRTR
DOWN

XXXXX

AdeJ(DWN) BamHI F AdeJ qRTF

adeJ

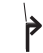

$\checkmark$ ade

AdeJ qRTR

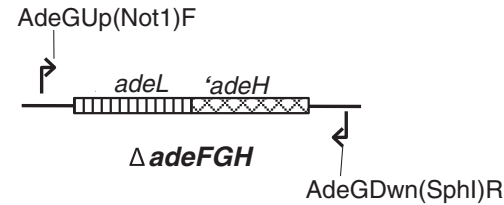

AdeGDwn(Sphl)R

Figure 1 (See legend on next page.) 
(See figure on previous page.)

Figure 1 Strategy for deleting adeL-adeFGH and adelJK operons in MDR A. baumannii DB and R2. Panel A, The upstream (UP) and downstream (DOWN) regions (approximately $1 \mathrm{~kb}$ ) flanking the target genes was cloned into the suicide vector, pMo130-Tel ${ }^{R}$. pMo130-Tel ${ }^{R}$ was constructed by inserting a 3.26 kb Xmal-digested tellurite-resistance cassette from pwFRT-Tel ${ }^{R}$ into the Xmal site of pMo130. Recombinants obtained after first cross-over were selected for inheritance of tellurite-resistance and $x y / E^{+}$(yellow colonies). These recombinants also do not produce any amplimers with the primer pair pMo130Tel F and pMo130Tel R. During the second cross-over, mutants with gene deletion (1) were selected for loss of $s a c B$ by passaging the first cross-over recombinants in media containing sucrose. The second cross-over could also yield parental genotype (2). Deletion of the adeFGH operon (Panel B) and the adelJK operon (Panel $\mathbf{C}$ ) showing the positions of the respective UP and DOWN fragments flanking each deletion (striped and hatched boxes, respectively). The locations of the PCR primers used for amplifying the UP and DOWN fragments and for qRT-PCR analysis of gene expression are indicated by black arrows while P1, P2 and P3 (grey arrows) are the locations of predicted promoters for adeFGH operon, adeL, and adelJK operon, respectively.

replacement. Such bacteria, which were white when sprayed with $0.45 \mathrm{M}$ pyrocathechol and were susceptible to $30 \mathrm{mg} / \mathrm{L}$ tellurite, usually appeared after the second passage. If the desired gene deletion had occurred, PCR of genomic DNA from these bacteria would produce only a $2 \mathrm{~kb}$ amplimer with the primer pair AdeGUp(Not1)F and AdeGDwn(Sph1)R. The same genomic DNA would not give any amplimer using the primer pair: AdeG RTF and AdeG RTR which annealed to the DNA that has been deleted (Figure 1B).

The suicide plasmid for deleting the adeIJK operon was constructed as described above but by first ligating the $1 \mathrm{~kb}$ UP fragment and a $0.9 \mathrm{~kb}$ DOWN fragment flanking the deletion before inserting into the pMo130TelR vector (Figure 1C). The UP and DOWN fragments were amplified from R2 genomic DNA using the primer pairs, AdeJ(UP) PstI F and AdeJ(UP)BamHI R, and AdeJ (DWN)BamHI F and AdeJ(DWN)SphI R, respectively (Figure $1 C$ and Additional file 1: Table S1). The UP and DOWN fragments were digested with $B a m \mathrm{HI}$ and ligated together in a 1:1 ratio. The ligated product was amplified using AdeJ(UP) PstI and AdeJ(DWN)SphI R to give a $1.9 \mathrm{~kb}$ amplimer which was then digested with Pst I and SphI and ligated with pMo130-Tel ${ }^{\mathrm{R}}$ linearized with PstI and SphI to give pMo130-Tel ${ }^{\mathrm{R}}$-adeJ(Up/Down). The plasmid construct was introduced into E. coli S17-1 and used for the two-step selection for deletion of the adeIJK operon as described above.

\section{Verification of genomic deletions}

Genomic deletions of the adeFGH and adeIJK operons in the mutants were verified by comparing the PCR amplimers obtained from the parental isolates and corresponding pump gene deletion mutants. For the pump gene deletions, PCR using primers flanking the deletion produced a 2-kb amplimer corresponding to the UP and DOWN fragments (Figure 2, lanes 3, 7, 11, 15, 17, 19, 21 and 23) while a larger wild-type amplimer was obtained using genomic DNA from the parental isolates, R2 and DB (Figure 2, lanes 1, 5, 9 and 13). For the $\triangle a d e F G H$ constructs, the deletion was also confirmed using PCR primers that annealed to the deleted region in adeG, whereby a 474 bp amplimer was obtained using genomic DNA from parental isolates (Figure 2, lanes 2 and 6), but no amplimer was obtained using genomic DNA from the $\triangle a d e F G H$ deletion mutants (Figure 2, lanes 4, 8,18 and 22). For the $\triangle a d e I J K$ constructs, the deletion produced a $0.26-\mathrm{kb}$ amplimer using the primers AdeJ $\mathrm{F}$ and AdeK R and genomic DNA from the $\triangle$ adeIJK mutants (Figure 2, lanes 12, 16, 20 and 24) and a longer 3.7-kb amplimer with genomic DNA from the wild-type parental isolates (Figure 2, lanes 10 and 14).

\section{Transcriptional analysis of the $\triangle a d e F G H$ and $\triangle$ adelJK deletion mutants}

RNA was extracted from parental strains and pump mutants cultured during mid-logarithmic growth in the absence of antibiotics. Analysis of the transcripts of the three major RND pumps in A. baumannii showed that the expression pattern of adeB, adeG and adeJ genes in both DB and R2 was similar (Figure 3). In the absence of any antibiotics, adeIJK was the most highly expressed pump while the expression of adeFGH was the lowest. All three pumps were also about 4-fold more highly expressed in DB as compared to R2 (Figure 3).

To confirm that the gene deletions had abolished the expression of the efflux pumps, the levels of transcripts of each gene in the adeFGH and adeIJK operons were measured in the deletion mutants and compared with the corresponding transcript levels in the parental strains. Both the DBDadeFGH and R2 $\triangle$ adeFGH mutants showed significant reduction (to $\leq 10 \%$ ) in the transcript levels for adeF, adeG and adeH when compared to the parental strains (Figure 4A). Although detectable, the level of adeL transcription in these mutants was also significantly reduced when compared to the adeL transcripts in the parental strains. This was because the genomic deletion had included the putative adeL promoter. Inactivation of adeG in both $\mathrm{DB} \triangle a d e F G H$ and R2 $\triangle$ adeFGH mutants was confirmed by the almost undetectable levels of adeG transcripts (Figure 4A).

Successful inactivation of adeJ was also similarly confirmed by the absence of adeJ transcripts in the DB $\triangle$ adeIJK and R2 $\Delta$ adeIJK mutants (Figure 4B). A small 


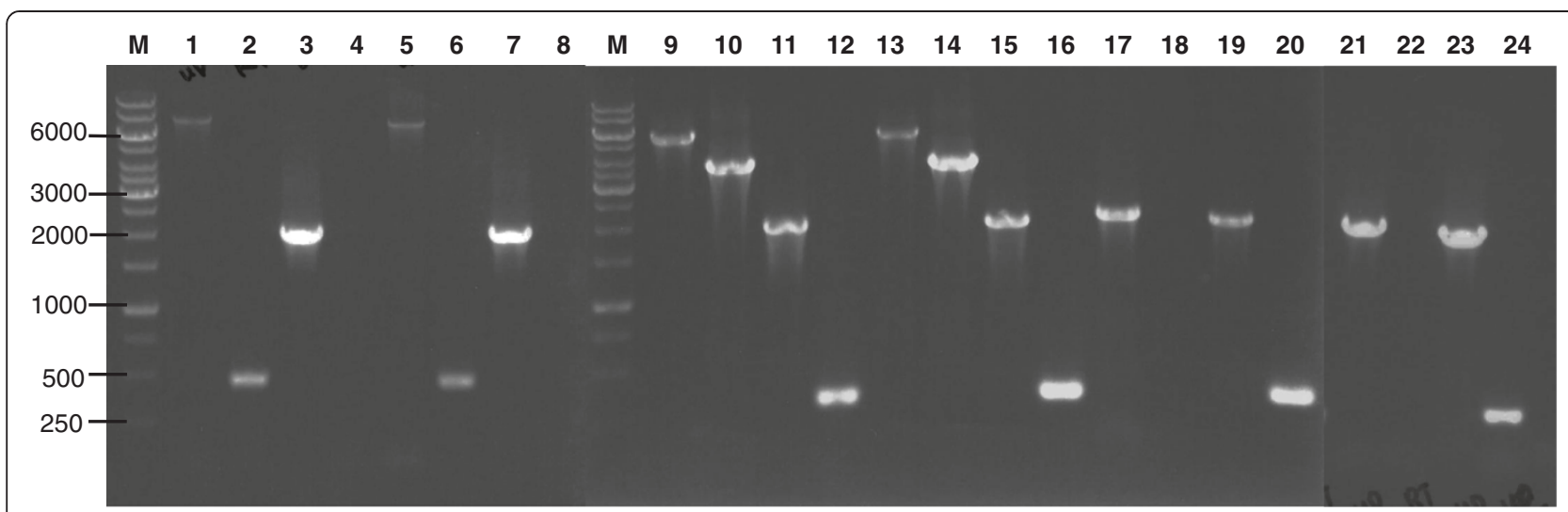

Figure 2 Verification of genomic deletions by PCR of genomic DNA. Amplimers using AdeGUp (Notl)F and AdeGDwn(Sphl)R for DB (lane 1)

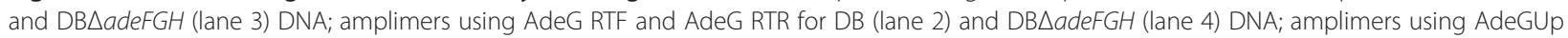
(Notl)F and AdeGDwn(Sphl)R for R2 (lane 5) and R2 $\triangle$ adeFGH (lane 7) DNA; amplimers using AdeG RTF and AdeG RTR for R2 (lane 6) and R2 $\triangle$ adeFGH (lane 8) DNA; amplimers using AdeJ(UP) Pstl F and AdeJ(DWN) Sphl R for R2 (lane 9), R2 $\triangle$ adelJK (lane 11), DB (lane 13) and DB $\triangle$ adelJK (lane 15); amplimers using AdeJ F and AdeK R for R2 (lane 10), R2 $\triangle$ adelJK (lane 12), DB (lane 14) and DB $\triangle$ adelJK (lane 16); DB $\triangle$ adeFGH $\triangle$ adelJK DNA amplified using AdeGUp (Notl)F and AdeGDwn(Sphl)R (lane 17), AdeG RTF and AdeG RTR (lane 18); AdeJ(UP) Pstl F

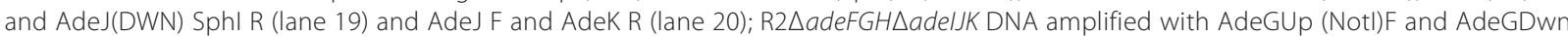
(Sphl)R (lane 21), AdeG RTF and AdeG RTR (lane 22), AdeJ(UP) Pstl F and AdeJ(DWN) Sphl R (lane 23) and AdeJ F and AdeK R (lane 24); M, 1 kb DNA ladder (GeneRuler ${ }^{T M}$ ).

quantity of adeI transcripts was udetectable in DB $\triangle$ adeIJK and R2 $\triangle$ adeIJK mutants, albeit at 56\% and $31 \%$ of wild-type levels, respectively. This was due to the location of the adeI qRT-PCR primers within the UP fragment, i.e. within the 5 ' undeleted portion of the adeI gene (Figure $1 \mathrm{C}$ ).

Next, we tested the feasibility of our marker-less deletion strategy for creating isogenic mutants carrying a combination of pump gene deletions. We applied this strategy to delete adeIJK in the DB $\triangle a d e F G H$ and R2 $\triangle a d e F G H$ mutants

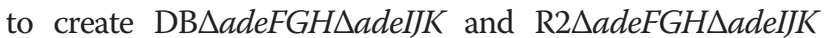
mutants, respectively. As expected, the DB $\triangle$ adeFGH$\Delta$ -

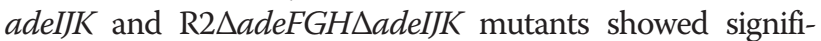
cantly reduced expression of adeL, adeF, adeG, adeH, adeJ

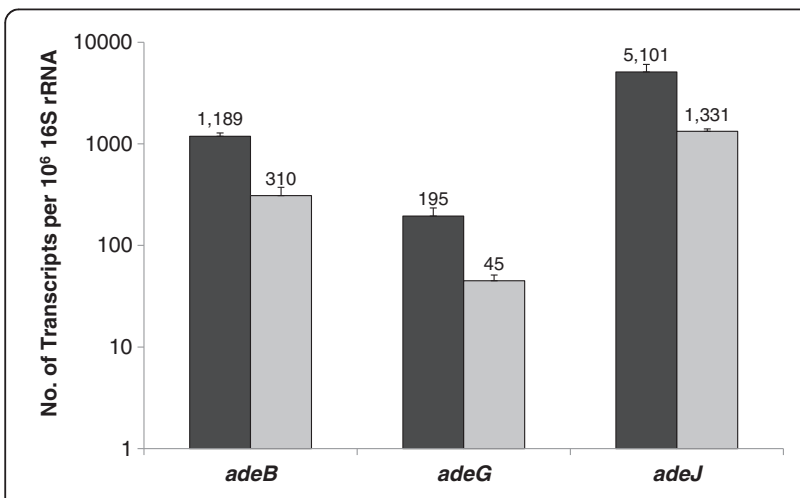

Figure 3 Relative expression of $a d e B$, adeG and adeJ in DB and R2 during mid-log phase. RNA was extracted from mid-log phase bacteria $\left(\mathrm{OD}_{600}=1.0\right)$ cultured in $\mathrm{LB}$ medium. The numbers of adeB, ade $G$ and adeJ transcripts were each normalized to 165 rRNA transcripts. Black bars, DB; Light grey bars, R2. and adeK (Figure 4C). Expression of adeI in DBDadeFGHA-

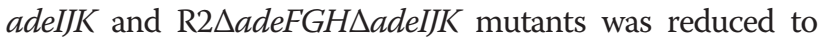
$38 \%$ and $58 \%$ of DB and R2 levels, respectively.

\section{Antimicrobial susceptibility profiles of pump deletion mutants}

The parental isolates, $\mathrm{DB}$ and $\mathrm{R} 2$, were MDR including to quinolones (nalidixic acid), fluoroquinolones (ciprofloxacin), chloramphenicol, tetracycline, carbapenems (meropenem and imipenem), $\beta$-lactams (piperacillin, oxacillin), cephalosporins (ceftazidime), macrolides (erythromycin), lincosamides (clindamycin), trimethoprim and aminoglycosides (gentamicin and kanamycin) (Table 1). Inactivation of the adeIJK in isolates DB and $\mathrm{R} 2$ resulted in at least a 4-fold increased susceptibility to nalidixic acid, chloramphenicol, clindamycin, tetracycline, minocycline and tigecycline, but had no effect on antimicrobial susceptibility to $\beta$-lactams (oxacillin and piperacillin), cephalosporins (ceftazidime), fluoroquinolones (ciprofloxacin), carbapenems (meropenem and imipenem), erythromycin and aminoglycosides (gentamicin and kanamycin). DB $\Delta$ adeIJK and R2 $\Delta$ adeIJK mutants were also 8-fold more susceptible to trimethoprim when compared to the parental isolates.

Although adeL and the adeFGH operon were expressed in $\mathrm{DB}$ and R2, albeit at a lower level that $a d e B$ and adeJ, inactivation of adeFGH in both $\mathrm{DB}$ and R2 had minimal impact on the MDR phenotype of DB and R2 (Table 1). This is shown by the minimal change in antimicrobial susceptibility between the mutants that had only adeFGH inactivated (DB $\triangle a d e F G H$ and $\mathrm{R} 2 \triangle a d e F G H$ ) and both adeFGH and adeIJK operons inactivated (DB $\triangle a d e F G H$ $\Delta$ adeIJK and R2 $\Delta$ adeFGH $\Delta$ adeIJK) (Table 1). The 


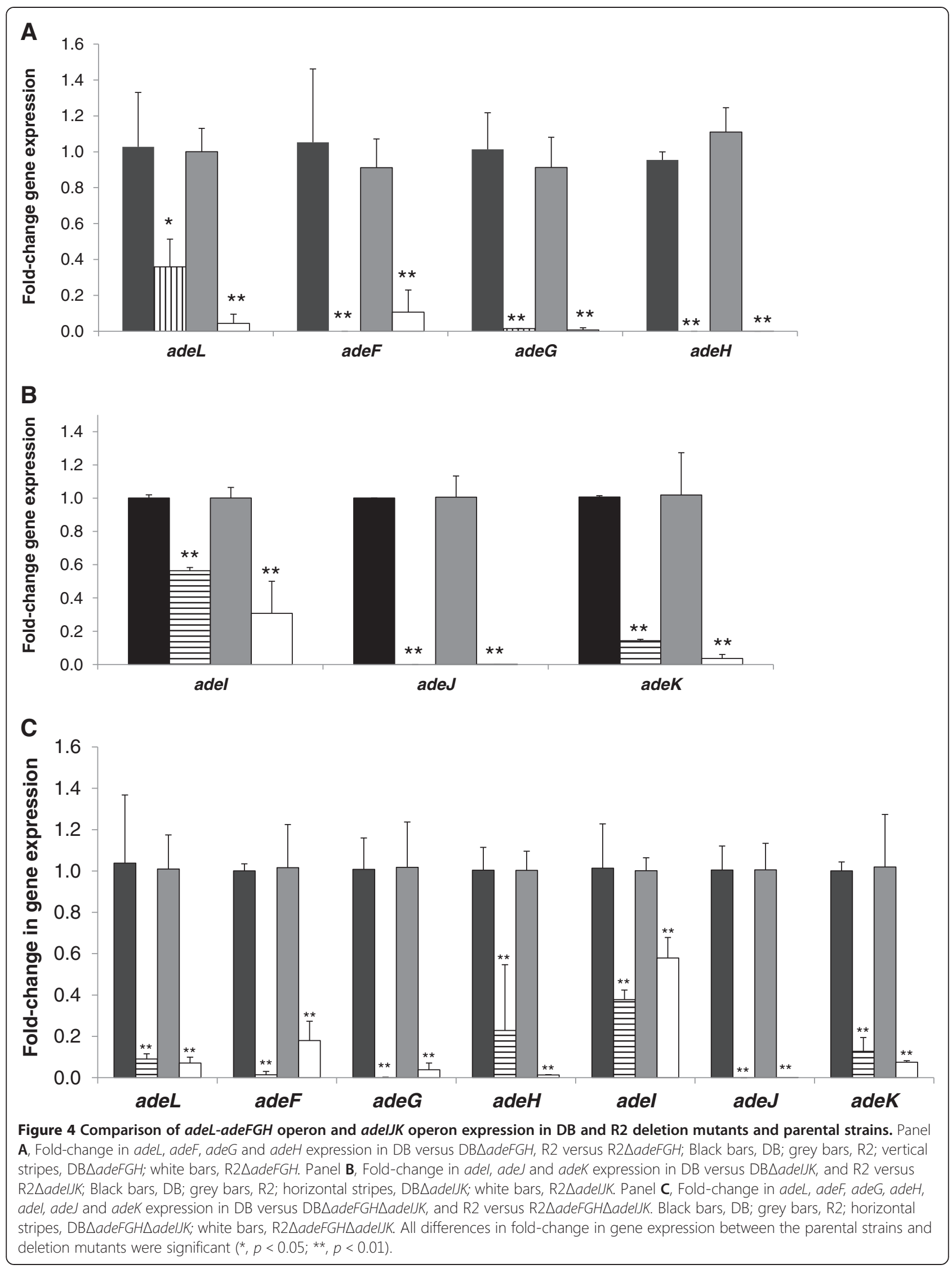


Table 1 Antimicrobial susceptibility of MDR A. baumannii DB, R2 and pump deletion mutants

\begin{tabular}{|c|c|c|c|c|c|c|c|c|}
\hline Antibiotic & DB & DBDadeFGH & DBAadelJK & DBDadeFGH $\Delta$ adelJK & $\mathrm{R} 2$ & R2 $\triangle$ adeFGH & R2DadelJK & R2 $\Delta a d e F G H \Delta a d e l J K$ \\
\hline Nalidixic acid & 512 & 512 & 128 & 128 & 1024 & 1024 & 256 & 256 \\
\hline Ciprofloxacin & 64 & 64 & 64 & 64 & 128 & 128 & 128 & 128 \\
\hline Chloramphenicol & 64 & 64 & 16 & 16 & 128 & 128 & 32 & 32 \\
\hline Tetracycline & 512 & 512 & 128 & 128 & 512 & 512 & 128 & 128 \\
\hline Minocycline & 2 & 2 & 0.25 & 0.25 & 2 & 2 & 0.5 & 0.5 \\
\hline Tigecycline & 1 & 1 & 0.25 & 0.25 & 1 & 1 & 0.25 & 0.25 \\
\hline Meropenem & 128 & 128 & 128 & 128 & 64 & 64 & 64 & 64 \\
\hline Imipenem & 32 & 32 & 32 & 32 & 64 & 64 & 64 & 64 \\
\hline Piperacillin & 512 & 512 & 512 & 512 & 256 & 256 & 256 & 256 \\
\hline Oxacillin & $>1024$ & $>1024$ & $>1024$ & $>1024$ & 1024 & 1024 & 1024 & 1024 \\
\hline Ceftazidime & 256 & 128 & 256 & 256 & 256 & 128 & 512 & 512 \\
\hline Erythromycin & 512 & 512 & 512 & 512 & 512 & 512 & 512 & 512 \\
\hline Clindamycin & 128 & 128 & 16 & 16 & 128 & 128 & 16 & 16 \\
\hline Trimethoprim & 128 & 128 & 16 & 16 & 128 & 128 & 16 & 16 \\
\hline Gentamicin & $>1024$ & $>1024$ & $>1024$ & $>1024$ & $>1024$ & $>1024$ & $>1024$ & $>1024$ \\
\hline Kanamycin & $>1024$ & $>1024$ & $>1024$ & $>1024$ & $>1024$ & $>1024$ & $>1024$ & $>1024$ \\
\hline
\end{tabular}

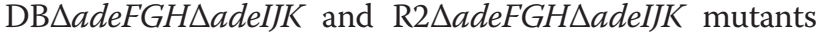
had the same antimicrobial susceptibility as DB $\triangle a d e I J K$ and R2 $\triangle$ adeIJK mutants, respectively (Table 1 ).

\section{Growth of pump deletion mutants}

The optical density at $600 \mathrm{~nm}$ measurements of liquid cultures of the parental strains and pump deletion mutants revealed no significant difference in growth kinetics (data not shown). Growth kinetics in the presence of sub-MIC concentrations of EIs were also carried out to simulate conditions in the H33342 accumulation assay (see below) and to ensure no inhibition of growth over a two-hour time period during the assay. These experiments showed that $30 \mathrm{mg} / \mathrm{L} \mathrm{CCCP} \mathrm{and} 50 \mathrm{mg} / \mathrm{L} \mathrm{PA} \beta \mathrm{N}$ did not restrict growth of R2 (data not shown). Viability of all strains was unaffected by $\mathrm{H} 33342$ concentrations of $2.5 \mu \mathrm{M}, 5 \mu \mathrm{M}$ and $10 \mu \mathrm{M}$ (data not shown).

\section{Accumulation of H33342 by efflux pump gene deletion mutants}

Compared with the parental isolate, $\mathrm{R} 2$, there was a significant 0.8 fold change in the level of $\mathrm{H} 33342$ accumulated at steady state in R2 $\triangle a d e F G H$ (Figure $5 \mathrm{~A}$ ). Compared with the parental isolate, accumulation of H33342 was significantly increased in R2 $\mathrm{R}$ adeIJK and R2 $\Delta$ adeFGH $\triangle$ adeIJK, with a fold change of 1.18 and 1.16 respectively. The mutants created in isolate DB showed a different pattern of accumulation (Figure 5B). The level of H33342 accumulated at steady state was significantly higher in all three mutants, DBDadeFGH,

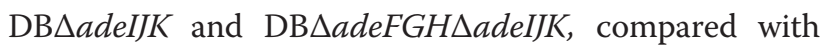
the parental strain, with fold-changes of 1.13, 1.26 and 1.22 , respectively.

Addition of CCCP caused a significant increase in the steady state accumulation of $\mathrm{H} 33342$ by all strains (Table 2). In the R2 isolate and mutants, this increase was most pronounced in $\mathrm{R} 2 \Delta a d e F G H$, with a fold increase of 1.46 observed (Table 2). The parental isolate showed a smaller fold increase of 1.31. R2 $\Delta$ adeIJK and R2 $\triangle$ adeFGH $\triangle$ adeIJK showed the smallest fold changes of 1.09 and 1.10, respectively. In the DB parent and mutant strain, the parental strain $\mathrm{DB}$ showed the highest fold increase of 1.51 after addition of CCCP, with the increase in DB $\triangle$ adeFGH slightly less, at 1.27 (Table 2). DB $\triangle a d e I J K$ and DB $\triangle a d e F G H \triangle a d e I J K$ again showed the smallest fold changes of 1.16 and 1.19, respectively. Addition of PA $\beta N$ also caused a significant increase in accumulation in all strains (Table 2). This increase was of a similar fold in the parental strains, $\mathrm{R} 2$ and DB, and their mutants.

\section{Accumulation of ethidium bromide by efflux pump gene deletion mutants}

It has been shown previously that H33342 and ethidium bromide are substrates of efflux pumps [11]. Therefore, accumulation of ethidium bromide was also measured. Compared with the parental isolate, the fold-change in the steady state levels of ethidium bromide accumulated in efflux pump mutants showed the same pattern as that produced with the H33342 accumulation assay, with 


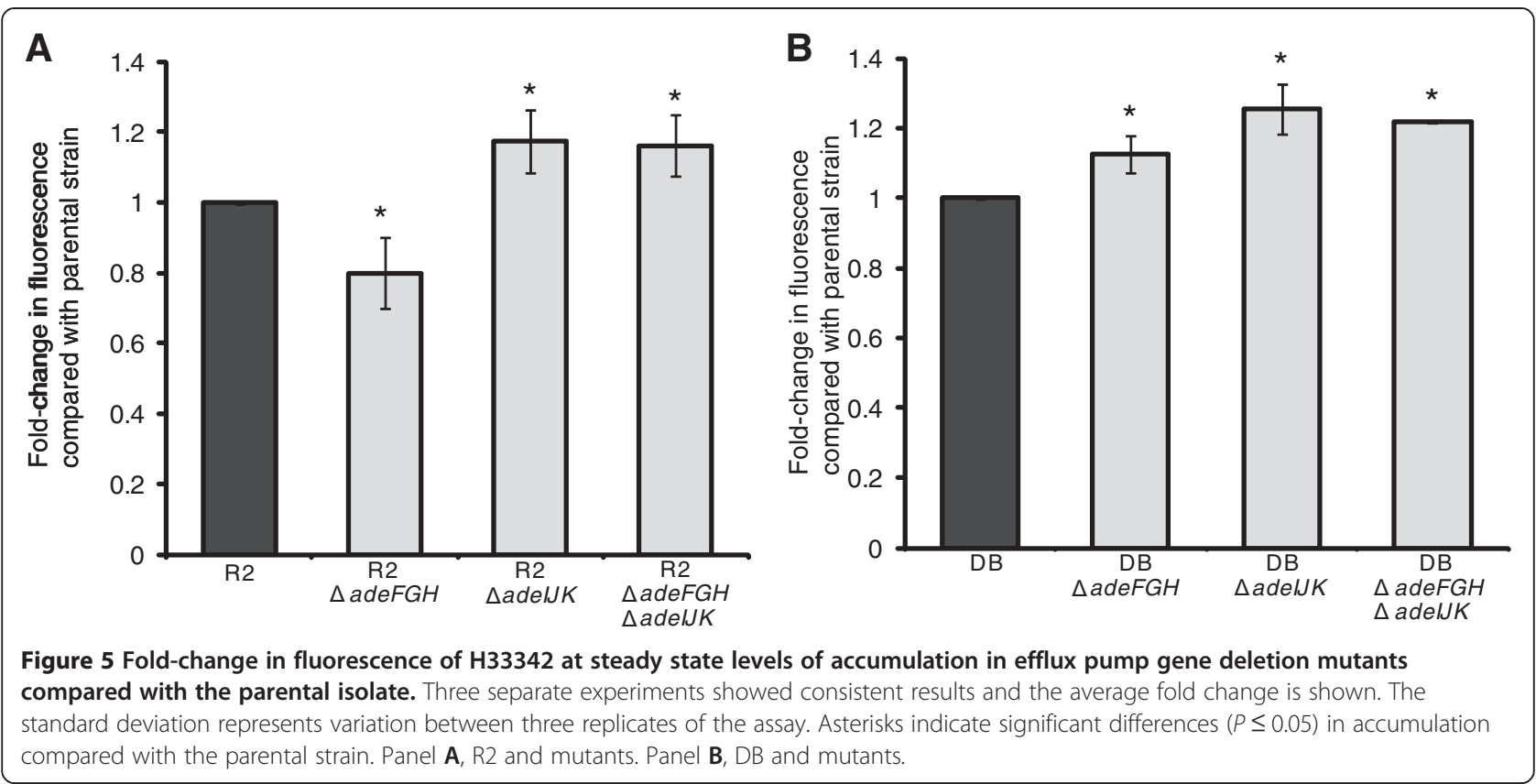

levels in R2 $\triangle a d e F G H$ significantly lower than in parental isolate R2 (Figure 6A), and R2 $\Delta a d e I J K$ and R2 $\triangle$ adeFGH$\triangle a d e I J K$ accumulating significantly higher levels. Efflux pump mutants DB $\triangle a d e F G H$, DB $\triangle a d e I J K$

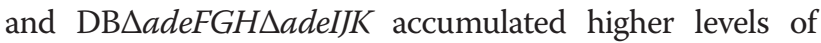
ethidium bromide than the parental isolate, DB (Figure 6C). Addition of both CCCP and PA $\beta \mathrm{N}$ produced a significant increase in the level of ethidium bromide accumulated at steady state in both parental isolates and their mutants and the effect was similar to that seen with H33342.

Table 2 Fold-change in fluorescence of H33342 at steady state level accumulation in the presence of Els in efflux pump mutants and parental strains

\begin{tabular}{|c|c|c|}
\hline Bacterial strain & $+\mathrm{CCCP}^{\mathrm{a}}$ & $+P A \beta N^{b}$ \\
\hline$\overline{D B}$ & $1.51 \pm 0.04$ & $1.29 \pm 0.11$ \\
\hline DB $\triangle$ adeFGH & $1.27 \pm 0.12$ & $1.28 \pm 0.03$ \\
\hline DB $\triangle$ adelJK & $1.16 \pm 0.06$ & $1.24 \pm 0.13$ \\
\hline 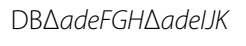 & $1.19 \pm 0.03$ & $1.36 \pm 0.07$ \\
\hline R2 & $1.31 \pm 0.12$ & $1.27 \pm 0.04$ \\
\hline R2 $\triangle$ adeFGH & $1.46 \pm 0.04$ & $1.29 \pm 0.03$ \\
\hline R2 $\triangle$ adelJK & $1.09 \pm 0.01$ & $1.29 \pm 0.05$ \\
\hline R2 $\triangle$ adeFGH $\triangle$ adelJK & $1.10 \pm 0.01$ & $1.20 \pm 0.10$ \\
\hline
\end{tabular}

Three separate experiments showed consistent results and representative examples are shown. The standard deviation represents variation between three biological replicates. All values shown are significant differences $(P \leq 0.05)$ in accumulation with addition of an El relative to absence of El. ${ }^{a}$ fold-change compared to corresponding bacterial sample in the absence of CCCP.

${ }^{b}$ fold-change compared to corresponding bacterial sample in the absence of PABN.

\section{Discussion}

The two-step deletion strategy we have described was used for creating unmarked deletions in the adeFGH and adeIJK efflux pump operons, separately and together, in two clinical MDR A. baumannii isolates. It is an improvement from the simple method for gene replacement in A. baumannii described by Aranda et al (2010) that uses an antibiotic resistance cassette [12]. To adapt the method first described for use in MDR $A$. baumannii, we introduced a tellurite resistance cassette into the pMo130 suicide vector created by Hamad et al (2009) to facilitate the selection of MDR A. baumannii transconjugants with the suicide plasmid inserted into the genome, i.e. first crossover products [8]. It was helpful to first ascertain the growth inhibitory concentration of tellurite for the parental A. baumannii strain so the number of transconjugants (first crossover) that are false positives can be minimized by using a suitable tellurite concentration. Passaging the first crossover recombinants in media containing sucrose provided the selection pressure for loss of the plasmid by a second crossover, leading to the formation of white colonies when sprayed with pyrocathechol.

The main advantage of this method, which does not use antibiotic selection for the gene deletion mutants, is its application for generating multiple gene deletions in a single strain as we have demonstrated by creating DB $\triangle a d e F G H \Delta a d e I J K$ and R2 $\triangle a d e F G H \triangle a d e I J K$ mutants. This is particularly important because the majority of $A$. baumannii strains are MDR or extensively drugresistant (XDR). Other than the MDR strains described in this study, we have also tested this method in a 


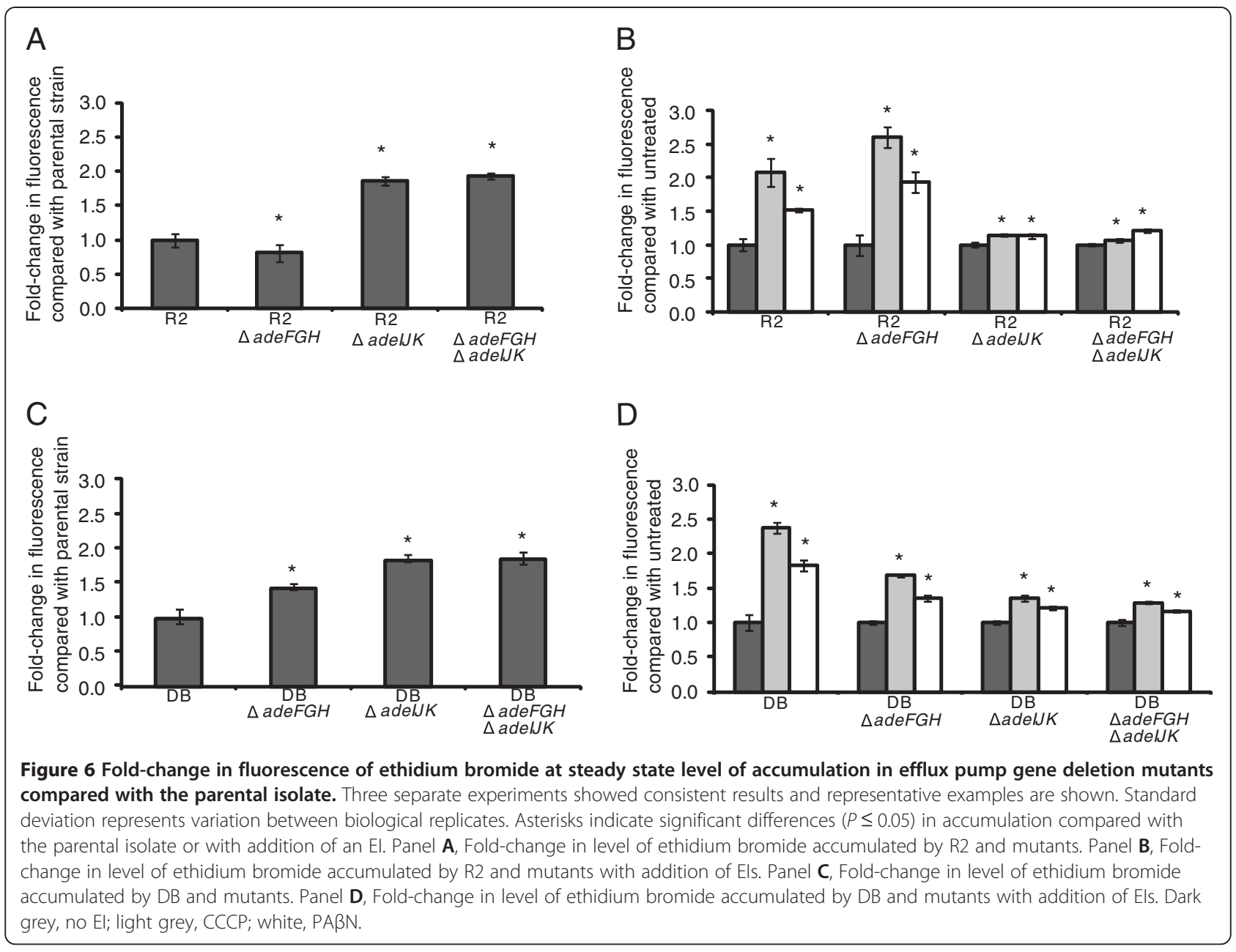

carbapenem-susceptible $A$. baumannii strain (data not shown). Un-marked deletion mutants are especially useful for ascertaining the contribution of each efflux pump to MDR as the presence of antibiotic resistance cassettes in the mutants may complicate the interpretation of antimicrobial susceptibility. We believe that the markerless method would allow the impact of each efflux system on antimicrobial resistance to be clearly defined.

The contribution of efflux pumps to antibiotic resistance in A. baumannii has been demonstrated with mutants created by gene inactivation/deletion or by creating spontaneous efflux pump overexpressing mutants via selection on antibiotic gradients, but with some inconsistencies in antimicrobial susceptibilities depending on how the genes were inactivated [5]. For example, inactivation of ade $A B C$ in a clinical MDR isolate by insertion of a ticarcillin-resistance gene conferred increased susceptibility to aminoglycosides, $\beta$-lactams, fluoroquinolones, chloramphenicol, tetracycline, macrolides and trimethoprim [7]. However when ade $A B C$ was deleted and an apramycin resistance cassette was inserted in the same MDR isolate, the $\triangle a d e A B C$ mutant showed increased susceptibility to fluoroquinolones, chloramphenicol, tetracycline, tigecycline and macrolides but no change in susceptibility to aminoglycosides, trimethoprim and $\beta$-lactams $[4,6]$. We hypothesized that the antibiotic resistance gene used in the creation of pump gene mutants complicated the interpretation of antimicrobial susceptibility data and hence which agents were putative substrates of each $A$. baumannii efflux pump.

When adeIJK was inactivated using the marker-less method, the MDR isolates became more susceptible to nalidixic acid, chloramphenicol, clindamycin, tetracycline, minocycline, tigecycline and trimethoprim. It is in-

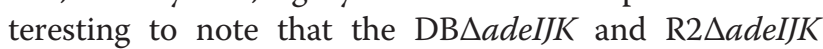
mutants showed increased susceptibility to nalidixic acid without affecting susceptibility to ciprofloxacin, suggesting AdeIJK may be specific for quinolones but not fluoroquinolones. We also noted that, although the AdeIJK pump confers increased resistance to exactly the same antibiotics in both DB and R2, the host genotype had an influence on the magnitude of resistance to each antibiotic. The successful creation of adeFGH and adeIJK gene deletions, separately and together, in two 
MDR A. baumannii isolates demonstrates the robustness of the method and its application across different MDR A. baumannii isolates. The antibiotic substrates revealed with our mutants are in general agreement with those described by Damier-Piolle et al (2008) in which adeIJK was inactivated in an MDR isolate by gene deletion together with insertion of a kanamycin-resistance cassette [6]. However, in our study the DBDadeIJK and R2 2 adeIJK mutants were also more susceptible to trimethoprim, but not to $\beta$-lactams. It should be noted that differences between these studies may be due to the presence of different antibiotic resistance genes on the host genome, e.g. R2 had bla $a_{O X A-23}$ like, bla $a_{O X A-51}$ like genes, bla $a_{T E M}$, bla $a_{O X A}$ and $b l a_{A D C}$ that confer $\beta$-lactam resistance. The MICs of antibiotics for double mutants

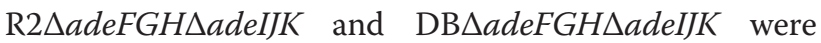
the same as for the corresponding single mutants $\mathrm{R} 2 \Delta a d e I J K$ and DB $\Delta a d e I J K$. This was expected, as a single deletion of adeFGH had minimal effect on MICs of antibiotics in either strain.

Deletion of adeIJK and of adeFGH in combination with adeIJK in both $\mathrm{R} 2$ and $\mathrm{DB}$ resulted in a significant increase in steady state accumulation levels of both H33342 and ethidium bromide. This infers reduced efflux in these strains, presumably as a consequence of the removal of the efflux pump AdeIJK. Addition of CCCP to $\triangle a d e I J K$ and $\triangle a d e F G H \triangle a d e I J K$ mutants of both R2 and DB significantly increased the steady state accumulation of H33342, suggesting that, despite lacking AdeIJK, these mutants still possess proton gradient dependent efflux activity as a result of another pump system. The addition of CCCP and PA $\beta N$ had the same effect on the accumulation of ethidium bromide. However, the increase in accumulation observed in these mutants was not as high as that seen with the parental isolates and the adeFGH deletion mutants, supporting the previous finding that efflux is reduced in mutants lacking adeIJK.

In our study, the deletion of the adeFGH operon also removed the putative adeL promoter, resulting in reduced expression of adeL. However, both the inactivation of the adeFGH operon and reduced expression of adeL had very little impact on antimicrobial susceptibility when compared to the parental isolates which expressed both adeL and adeFGH operon. This was also true when the antimicrobial susceptibilities of DB and R2 mutants that had both the adeIJK and adeFGH operons deleted were compared with the DB and R2 mutants that had only the adeIJK operon inactivated. In all instances, inactivation of adeFGH had minimal impact on antimicrobial susceptibility when compared to isogenic isolates with functional AdeFGH, indicating that expression of adeL and adeFGH operon was not involved in the multidrug resistance of these clinical MDR isolates. These findings are different to those of Coyne et al, who showed that overexpressing adeFGH in an MDR strain lacking AdeABC and AdeIJK increased the MICs of several antibiotics including chloramphenicol, clindamycin, tetracycline, minocycline, tigecycline, norfloxacin, ciprofloxacin and cotrimoxazole [5]. In that study, the adeFGH operon was overexpressed in a spontaneous drug-resistant $\triangle a d e A B C \triangle a d e I J K$ mutant selected on norfloxacin and chloramphenicol gradient plates. The adeFGH operon was then deleted and a streptomycin-spectinomycin resistance cassette was also inserted to select for the deletion mutant. It is plausible that the process of selecting spontaneous drug-resistant mutants on chloramphenicol and norfloxacin gradients may have created gene duplication and amplification or a mutation in another efflux pump regulator was selected, especially since the inhibition of DNA gyrase by fluoroquinolones induces the SOS response [13]. It is also possible that under the experimental conditions whereby the adeFGH operon was induced and significantly overexpressed, an increase in resistance to chloramphenicol, trimethoprim and clindamycin may be observed. However, further studies are needed to determine the significance and relevance of such conditions to the clinical environment [14]. It was proposed that mutation in adeL results in overexpression of adeFGH operon and hence an increase in antibiotic resistance [5]. It is also possible that mutation in adeL, a LysR-type transcriptional regulator, may affect expression of another efflux pump gene/s or antibiotic resistance determinant. However, in the DBAadeFGH and R2 $\triangle a d e F G H$ mutants created in the present study, adeL expression was impaired yet there was minimal change in the MICs of antibiotics for the mutants when compared with the parental isolates. This ruled out the possibility that the MDR phenotype of $\mathrm{DB}$ and $\mathrm{R} 2$ might be due to a mutation in adeL which had an effect on the expression of another efflux pump(s) other than the adeFGH operon. Our data suggests that the activities of the AdeL transcriptional regulator and AdeFGH pump do not contribute to multidrug resistance in DB and $\mathrm{R} 2$.

Despite the minimal change in MICs of antibiotics compared with the parental isolate, R2 $\triangle$ adeFGH showed a significant decrease in accumulation of both H33342 and ethidium bromide, inferring increased efflux in this strain. This may be due to increased expression of another efflux system in order to compensate for the loss of AdeFGH. This could also explain the lack of change in MIC seen with deletion of adeFGH. Previous work in Salmonella enterica serovar Typhimurium has shown that deletion of RND efflux pump genes can lead to compensatory altered expression of other efflux pump genes. For example, deletion of $a c r B$ in SL1344 resulted in a 7.9 fold increase in the expression of acrF [15]. An 
increase in accumulation of H33342 and ethidium bromide was seen in DB $\triangle$ adeFGH, inferring reduced efflux in this strain, however this difference did not translate into a change in MIC. Addition of CCCP and PA $\beta N$ had a greater effect on accumulation of $\mathrm{H} 33342$ and ethidium bromide in this efflux pump mutant than in mutants lacking adeIJK. A greater fold change in accumulation was seen with both R2 $\triangle a d e F G H$ and DB $\triangle a d e F G H$ than other efflux pump mutants, suggesting that efflux activity is higher in these mutants.

Using the marker-less deletion method, we have demonstrated that AdeFGH and AdeIJK are independent efflux pumps with no common antibiotic substrates. While both adeFGH and adeIJK operons are expressed in MDR A. baumannii, only the expression of adeIJK contributed to increased resistance to nalidixic acid, chloramphenicol, clindamycin, tetracycline, minocycline, tigecycline and trimethoprim. Expression of adeFGH was not the cause of resistance in the clinical isolates of MDR A. baumannii, DB and R2.

\section{Conclusions}

The marker-less gene deletion method we have described is useful for creating gene deletions in MDR $A$. baumannii. Deletions of the adeFGH and adeIJK efflux pump operons, separately and together, were created in two clinical MDR A. baumannii isolates to demonstrate the robustness of the method. Even though both adeFGH and adeIJK operons are expressed in MDR $A$. baumannii, only the expression of adeIJK contributed to increased resistance to nalidixic acid, chloramphenicol, clindamycin, tetracycline, minocycline, tigecycline and trimethoprim. Expression of adeFGH was not the cause of resistance in the clinical isolates of $\operatorname{MDR} A$. baumannii, DB and R2. This method allows the impact of each efflux system on antimicrobial resistance to be clearly defined.

\section{Methods}

Bacterial strains, plasmids and culture conditions

Bacterial strains and plasmids used in this study are listed in Table 3. Acinetobacter baumannii R2 (TTSH6013 654325/06) and DB (DB15354/07) were clinical isolates from a collection by the Network for Antimicrobial Resistance Surveillance, Singapore. According to the interim standard definitions for acquired resistance, both $\mathrm{DB}$ and $\mathrm{R} 2$ are classified as MDR as they are non-susceptible to $\geq 1$ agent in $\geq 3$ antimicrobial categories (aminoglycosides, fluoroquinolones, carbapenems, tetracycline, extended spectrum cephalosporins, folate pathway inhibitors) [17]. DB and R2 carry and express $b l a_{\text {OXA-23-like }}$ and $b l a_{\text {OXA-51-like, do not carry }}$

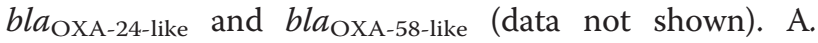
baumannii and E. coli were cultured under aerobic conditions at $37^{\circ} \mathrm{C}$ in Luria-Bertani Miller (LB) agar or LB broth (Becton Dickinson, Cockeysville, U.S.A.). Antibiotics used were at the following concentrations for $E$. coli: kanamycin, $10 \mathrm{mg} / \mathrm{L}$; tellurite $6 \mathrm{mg} / \mathrm{L}$; and for $A$. baumannii: tellurite, $30 \mathrm{mg} / \mathrm{L}$.

\section{DNA manipulations}

Bacterial genomic DNA was extracted using a rapid procedure described by Pitcher et al [18]. Plasmid DNA was extracted using GeneAid Hi-Speed Plasmid Mini kit (GeneAid, Taiwan). Standard PCR amplifications were performed with Biotools DNA polymerase (Biotools, Spain). All primers used for PCR were synthesized by $1^{\text {st }}$ Base Singapore and are listed in Additional file 1: Table $\mathrm{S} 1$. Electrocompetent cells were prepared from $6 \mathrm{ml}$ overnight bacterial culture according to the procedure described by Choi et al (2005) [19]. Electroporation was carried out by placing $100 \mu \mathrm{l}$ electrocompetent cells and $3 \mu \mathrm{l}$ plasmid DNA in a sterile cuvette $(0.1 \mathrm{~cm}$ electrode gap, Bio-Rad) and pulsed at $1.8 \mathrm{~V}$ using settings for bacteria in a Bio-Rad MicroPulser.

The plasmid, pwFRT-Tel ${ }^{\mathrm{R}}$, was digested with $X m a I$ and the $3.265 \mathrm{~kb}$ fragment carrying the tellurite-resistance cassette was isolated and ligated with XmaI-linearized pMo130 to produce the suicide plasmid, pMo130-Tel ${ }^{R}$. The orientation of the tellurite-resistance cassette insert shown in Figure 1A was ascertained by digesting the plasmid with Xho1 and BamHI which gave a $4.161 \mathrm{~kb}$ and a $5.231 \mathrm{~kb}$ band. An insertion of the tellurite-resistance cassette into pMo130-Tel ${ }^{\mathrm{R}}$ in the opposite orientation would have produced two bands of $1.150 \mathrm{~kb}$ and $8.242 \mathrm{~kb}$.

\section{Conjugative transfer}

E. coli S17-1 donor strain harboring the respective pMo130-TelR-(Up/Down) constructs and the A. baumannii recipient strains were cultured overnight at $37^{\circ} \mathrm{C}$ in $2 \mathrm{ml}$ LB (supplemented with kanamycin for the donor $E$. coli strain). Aliquots of $0.2 \mathrm{ml}$ each of donor and recipient cells were added to a microfuge tube containing $1.2 \mathrm{ml}$ of $\mathrm{LB}$ and washed twice with $2 \mathrm{ml} \mathrm{LB}$ each time. The cells were then suspended in $30 \mu \mathrm{l} \mathrm{LB}$ medium and added on to a sterile $0.45 \mu \mathrm{m}$ cellulose nitrate filter paper (Sartorius Stedim, NY, U.S.A.) on LB agar and incubated at $30^{\circ} \mathrm{C}$ for $16 \mathrm{~h}$. The cells were washed off from the filter by adding $0.4 \mathrm{ml}$ of $0.9 \% \mathrm{NaCl}$. Aliquots of $0.1 \mathrm{ml}$ were plated onto LB agar containing tellurite $(30 \mathrm{mg} / \mathrm{L})$ and gentamicin $(25 \mathrm{mg} / \mathrm{L})$ and incubated at $37^{\circ} \mathrm{C}$ for at least $16 \mathrm{~h}$. Gentamicin was added for counter-selection against the donor cells.

\section{RNA analysis and quantitative real-time PCR (qRT-PCR)}

RNA was extracted from mid-log phase bacteria prepared by inoculating $10 \mathrm{ml}$ Luria-Bertani (LB) broth Miller ( $1^{\text {st }}$ BASE Pte Ltd, Singapore) with an overnight culture (1:50) and incubating at $37^{\circ} \mathrm{C}$, with shaking at 
Table 3 List of bacterial strains and plasmids used in this study

\begin{tabular}{|c|c|c|}
\hline Strain or plasmid & Relevant characteristics & Reference or source \\
\hline \multicolumn{3}{|l|}{ A. baumannii strains } \\
\hline R2 & Wild-type clinical MDR isolate TTSH6013 624325/06 & $\begin{array}{l}\text { Network for Antimicrobial } \\
\text { Resistance Surveillance (Singapore) }\end{array}$ \\
\hline DB & Wild-type clinical MDR isolate DB15354/07 & $\begin{array}{l}\text { Network for Antimicrobial } \\
\text { Resistance Surveillance (Singapore) }\end{array}$ \\
\hline R2 $\triangle$ adeFGH & R2 with deletion in adeFGH operon & This study \\
\hline R2 $\triangle$ adelJK & R2 with deletion in adelJK operon & This study \\
\hline R2 $\triangle$ adeFGHDadelJK & R2 with deletion in adeFGH and adelJK operons & This study \\
\hline $\mathrm{DB} \triangle a d e F G H$ & DB with deletion in adeFGH operon & This study \\
\hline DBDadelJK & DB with deletion in adelJK operon & This study \\
\hline 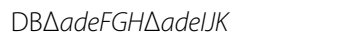 & DB with deletion in adeFGH and adelJK operons & This study \\
\hline \multicolumn{3}{|l|}{ E. coli strains } \\
\hline $\mathrm{DH} 5 \mathrm{a}$ & $\begin{array}{l}\text { F- Ф80lacZDM15 } \Delta \text { (lacZYA-argF) U169 recA1 endA1 hsdR17 } \\
\text { phoA supE44 } \lambda \text { - thi-1 gyrA96 relA1 }\end{array}$ & Invitrogen \\
\hline S17-1 & Genotype: recA pro hsdR RP4-2-Tc::Mu-Km::Tn7, Gm & {$[16]$} \\
\hline \multicolumn{3}{|l|}{ Plasmids } \\
\hline pMo130 & Suicide plasmid, $x y / E^{+}, s a c B^{+}, \mathrm{Km}^{R}$ & {$[8]$} \\
\hline pwFRT-Tel ${ }^{R}$ & Donor of tellurite resistance cassette & {$[10]$} \\
\hline pMo130-Tel ${ }^{R}$ & 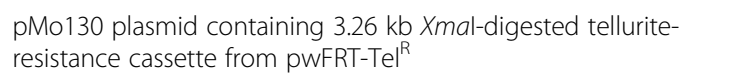 & This study \\
\hline pMo130-Tel' - -P8(UP/DWN) & $\begin{array}{l}\text { pMo130-Tel }{ }^{R} \text { containing a } 1 \mathrm{~kb} \text { UP fragment (promoterless adeL) } \\
\text { and } 1 \mathrm{~kb} \text { DOWN fragment ( } 3^{\prime} \text { partial adeH) }\end{array}$ & This study \\
\hline pMo130-Tel ${ }^{R}$-adeJ(Up/Down) & $\begin{array}{l}\text { pMo130-Tel }{ }^{R} \text { containing a } 1 \mathrm{~kb} \text { UP fragment (5' partial adel) } \\
\text { and } 0.9 \mathrm{~kb} \text { DOWN fragment (3' partial adeK) }\end{array}$ & This study \\
\hline
\end{tabular}

$120 \mathrm{rpm}$, until $\mathrm{OD}_{600}=1.0$. Triplicates of culture volumes containing two $\mathrm{OD}_{600}$ units $\left(\sim 2 \times 10^{9}\right.$ cells $)$ were centrifuged at 3,000 g for $10 \mathrm{~min}$ to harvest the cells. The cells were lysed by adding $1 \mathrm{~mL}$ of TRIzol $^{\circ}$ (Invitrogen, Carlsbad, CA) to the cell pellet and RNA was extracted according to the manufacturer's protocol. Contaminating DNA was removed by treating the RNA sample with Ambion TURBO $^{\mathrm{Tm}}$ DNase (Invitrogen) and cDNA was synthesized using random hexamer primers and TaqMan Reverse Transcription Reagents (Invitrogen) according to the manufacturer's protocol.

Primers for qRT-PCR of efflux pumps genes were designed using PrimerQuest (Integrated DNA Technologies, Coralville, IA) and are listed in Additional file 1: Table S2 (Supplementary files). qRT-PCR was performed using KAPA SYBR ${ }^{\circ}$ FAST Universal 2X qPCR Master Mix (Kapa Biosystems Inc., Woburn, MA) using 1X ROX (High) reference dye, $500 \mathrm{~nm}$ primers and $10 \mathrm{ng}$ cDNA in a total volume of $20 \mu \mathrm{L}$ and the transcripts were detected using Applied Biosystems 7300 Real-Time PCR system (Applied Biosystems, Carlsbad, CA). 16S rRNA was used for normalization of the qRT-PCR gene transcripts. qRT-PCR was performed twice for each of the triplicate RNA extracts. Data from each quantitative run was exported from the 7300 System software and analysed using $2^{-\Delta \Delta C t}$ calculations [20].

\section{Antimicrobial susceptibility testing}

Minimum inhibitory concentrations (MICs) of antibiotics were determined using the agar doubling dilution method according to BSAC standard methodology [21]. MICs of imipenem and meropenem were determined by E-test (Biomerieux, Hampshire, UK).

\section{Measurement of growth kinetics}

Bacterial strains were grown with aeration in LB broth at $37^{\circ} \mathrm{C}$ overnight. Bacterial cultures were diluted 1:100 in sterile Luria Bertani (LB) broth and $100 \mu \mathrm{l}$ of this suspension was added to each well of a clear 96 well microtitre tray. Optical density (OD) at an absorbance of $600 \mathrm{~nm}$ was measured over 16 hours in a BMG FLUOstar Optima (BMG, UK) at $37^{\circ} \mathrm{C}$. The BMG FLUOstar is sensitive to an OD600 of between 0.0 and 4.0 and reproducibility is \pm 0.010 for the OD range of $0.0-2.0$ (www.bmglabtech. com). Each experiment included three biological replicates and three technical replicates of each bacterial strain. Differences in generation times and final OD at $600 \mathrm{~nm}$ were calculated using a Student's $t$-test. $P$ values $\leq 0.05$ were considered as significant.

For assessment of toxicity of EIs and H33342, bacterial strains were grown with aeration in $\mathrm{LB}$ broth at $37^{\circ} \mathrm{C}$ overnight. A $4 \%$ inoculum $(120 \mu \mathrm{l}$ in $3 \mathrm{ml})$ of bacterial 
culture was added to fresh LB broth. This suspension was incubated with aeration at $37^{\circ} \mathrm{C}$ until the culture reached an OD at $600 \mathrm{~nm}$ of $0.6\left(=10^{8} \mathrm{cfu} / \mathrm{ml}\right)$. Cells were harvested by centrifugation at $2200 \mathrm{~g}$ for $10 \mathrm{~min}$ at room temperature and resuspended in $3 \mathrm{ml}$ sterile LB broth at room temperature. The OD at $600 \mathrm{~nm}$ of the suspension was measured and adjusted to 0.5 to standardize the number of bacterial cells in each culture and to simulate the conditions used in the H33342 accumulation assay. The bacterial suspension $(196 \mu \mathrm{l})$ was added to each well of a clear 96 well microtitre tray, along with $4 \mu \mathrm{l}$ of EI and $20 \mu \mathrm{l} \mathrm{H33342}$ at the required concentrations (see Results). OD at an absorbance of $600 \mathrm{~nm}$ was measured over 16 hours in the BMG FLUOstar OPTIMA (BMG, UK) at $37^{\circ} \mathrm{C}$. Each experiment included three biological replicates and three technical replicates of each bacterial strain. Differences in generation times and final OD at $600 \mathrm{~nm}$ were calculated using a Student's $t$-test. $P$ values $\leq 0.05$ were considered as significant.

\section{H33342 bis-benzamide accumulation in efflux pump deletion mutants}

Accumulation of H33342 was measured and data analysed as described previously [11]. The level at which maximum fluorescence was reached and remained unchanged within the time period of the assay was taken as the steady state accumulation level. The fold change in fluorescence of mutants compared to the parental clinical isolate in the presence and absence of efflux pump inhibitors (EI) was calculated. Student's $t$-tests were carried out to compare the accumulation of H33342 by the mutant with the parental strain, R2; $P$ values $<0.05$ were taken as significant. Each assay was repeated 3 times with 3 biological replicates.

\section{Ethidium bromide accumulation in efflux pump deletion mutants}

Ethidium bromide assays were carried out in the same way as the H33342 accumulation assay, except that cultures were resuspended in $1 \mathrm{M}$ sodium phosphate buffer with $5 \%$ glucose. A $1 \mathrm{mM}$ ethidium bromide stock solution was prepared and $20 \mu \mathrm{l}$ was injected to give a final concentration of $0.1 \mathrm{mM}$ in the assay. Fluorescence was measured over 117 minutes at excitation and emission wavelengths of $530 \mathrm{~nm}$ and $600 \mathrm{~nm}$, respectively, in a FLUOstar OPTIMA.

\section{Additional file}

Additional file 1: Table S1. Description of primers used for PCR and DNA sequencing. Table S2. List of primers used for quantitative real-time PCR.

\section{Authors' contributions}

IA and PS jointly constructed the adeFGH and adelJK deletion mutants and carried out the molecular genetic studies. GER performed the dye accumulation antimicrobial susceptibility assays. THK provided the MDR A. baumannii isolates, characterized the bla oxA sequences in DB and R2. KLC conceived the study. LJP and KLC participated in the design and coordination and helped to draft the manuscript. All authors read and approved the final manuscript.

\section{Acknowledgements}

We thank Martin Voskuil and Tung T. Hoang for their gifts of pMo130 and pwFRT-Tel ${ }^{R}$.

This work was supported by a Singapore-UK grant: A*STAR-UK MRC JGC1366/G0801977 and MRC grant DKAA RRAK 14525 to Laura Piddock.

\section{Author details}

${ }^{1}$ Department of Biochemistry, Yong Loo Lin School of Medicine, National University of Singapore, 8 Medical Drive, Singapore 117597, Singapore. ${ }^{2}$ Department of Pathology, Singapore General Hospital, Outram RoadSingapore169608. ${ }^{3}$ School of Immunity and Infection, College of Medical and Dental Sciences, University of Birmingham, Edgbaston, Birmingham B15 2TT, UK

Received: 17 April 2013 Accepted: 5 July 2013

Published: 13 July 2013

\section{References}

1. Visca P, Seifert $H$, Towner $\mathrm{KJ}$ : Acinetobacter infection-an emerging threat to human health. IUBMB Life 2011, 63(12):1048-1054.

2. Ho J, Tambyah PA, Paterson DL: Multiresistant Gram-negative infections: a global perspective. Curr Opin Infect Dis 2010, 23(6):546-553.

3. Durante-Mangoni E, Zarrilli R: Global spread of drug-resistant Acinetobacter baumannii: molecular epidemiology and management of antimicrobial resistance. Future Microbiol 2011, 6(4):407-422.

4. Coyne S, Courvalin P, Perichon B: Efflux-mediated antibiotic resistance in Acinetobacter spp. Antimicrob Agents Chemother 2011, 55(3):947-953.

5. Coyne S, Rosenfeld N, Lambert T, Courvalin P, Perichon B: Overexpression of resistance-nodulation-cell division pump AdeFGH confers multidrug resistance in Acinetobacter baumannii. Antimicrob Agents Chemother 2010, 54(10):4389-4393.

6. Damier-Piolle L, Magnet S, Bremont S, Lambert T, Courvalin P: AdelJK, a resistance-nodulation-cell division pump effluxing multiple antibiotics in Acinetobacter baumannii. Antimicrob Agents Chemother 2008, 52(2):557-562.

7. Magnet $\mathrm{S}$, Courvalin P, Lambert T: Resistance-nodulation-cell division-type efflux pump involved in aminoglycoside resistance in Acinetobacter baumannii strain BM4454. Antimicrob Agents Chemother 2001, 45(12):3375-3380.

8. Hamad MA, Zajdowicz SL, Holmes RK, Voskuil MI: An allelic exchange system for compliant genetic manipulation of the select agents Burkholderia pseudomallei and Burkholderia mallei. Gene 2009 430(1-2):123-131.

9. Sanchez-Romero JM, Diaz-Orejas R, De Lorenzo V: Resistance to tellurite as a selection marker for genetic manipulations of Pseudomonas strains. Appl Environ Microbiol 1998, 64(10):4040-4046.

10. Barrett AR, Kang Y, Inamasu KS, Son MS, Vukovich JM, Hoang TT: Genetic tools for allelic replacement in Burkholderia species. Appl Environ Microbiol 2008, 74(14):4498-4508.

11. Richmond GE, Chua KL, Piddock L: Efflux in Acinetobacter baumannii can be determined by measuring accumulation of $\mathrm{H} 33342$ (bis-benzamide). J Antimicrob Chemother 2013, 68:1594-1600.

12. Aranda J, Poza M, Pardo BG, Rumbo S, Rumbo C, Parreira JR, Rodriguez-Velo $P, B o u$ G: A rapid and simple method for constructing stable mutants of Acinetobacter baumannii. BMC Microbiol 2010, 10:279.

13. Blazquez J, Couce A, Rodriguez-Beltran J, Rodriguez-Rojas A: Antimicrobials as promoters of genetic variation. Curr Opin Microbiol 2012, 15:561-569.

14. Cortez-Cordova J, Kumar A: Activity of the efflux pump inhibitor phenylalanine-arginine beta-naphthylamide against the AdeFGH pump of Acinetobacter baumannii. Int J Antimicrob Agents 2011, 37(5):420-424.

15. Eaves DJ, Ricci V, Piddock LJ: Expression of acrB, acrF, acrD, marA, and soxS in Salmonella enterica serovar Typhimurium: role in multiple antibiotic resistance. Antimicrob Agents Chemother 2004, 48(4):1145-1150.

16. Andrews J: Determination of Minimum Inhibitory Concentrations. J Antimicrob Chemother Suppl 2001, 48(Suppl. S1):5-16.

17. Magiorakos AP, Srinivasan A, Carey RB, Carmeli Y, Falagas ME, Giske CG, Harbarth S, Hindler JF, Kahlmeter G, Olsson-Liljequist B, et al: Multidrug- 
resistant, extensively drug-resistant and pandrug-resistant bacteria: an international expert proposal for interim standard definitions for acquired resistance. Clin Microbiol Infect 2012, 18(3):268-281.

18. Simon R, Priefer U, Puhler A: A Broad Host Range Mobilization System for In Vivo Genetic Engineering: Transposon Mutagenesis in Gram Negative Bacteria. Nat Biotech 1983, 1(9):784-791.

19. Pitcher DG, Saunders NA, Owen RJ: Rapid extraction of bacterial genomic DNA with guanidium thiocyanate. Lett Appl Microbiol 1989, 8(4):151-156.

20. Choi KH, Kumar A, Schweizer HP: A 10-min method for preparation of highly electrocompetent Pseudomonas aeruginosa cells: application for DNA fragment transfer between chromosomes and plasmid transformation. J Microbiol Methods 2006, 64(3):391-397.

21. Livak KJ, Schmittgen TD: Analysis of relative gene expression data using real-time quantitative PCR and the 2(-Delta Delta $C(T))$ method. Methods 2001, 25(4):402-408.

doi:10.1186/1471-2180-13-158

Cite this article as: Amin et al:: A Method for generating marker-less gene deletions in multidrug-resistant Acinetobacter baumannii. BMC Microbiology 2013 13:158.

\section{Submit your next manuscript to BioMed Central and take full advantage of:}

- Convenient online submission

- Thorough peer review

- No space constraints or color figure charges

- Immediate publication on acceptance

- Inclusion in PubMed, CAS, Scopus and Google Scholar

- Research which is freely available for redistribution 